\title{
Flow and heat transfer in anisotropic active foam porous media wall
}

\author{
Rafael Deptulski ${ }^{1}$, Rachid Bennacer ${ }^{1, *}$ and Gisele Vieira ${ }^{2, *}$ \\ ${ }^{1}$ University Paris-Saclay, LMT-ENS 94230 Cachan, France \\ ${ }^{2}$ CEFET/RJ, DEMEC, Maracanã, Rio de Janeiro, 20271-110, Brazil
}

\begin{abstract}
The development of positive-energy buildings is nowadays an important societal challenge and the research objective of many laboratories. Working towards this direction, we describe in the present report the development of active and reactive walls, by employing a multi-fiber, volumetrically structured porous medium. Flow and transfer characterization in such a foam structure is not fully understood due to the strong nonlinearities included. These are amplified by heterogeneities and porosity changes, where the local and global flow affect the thermal field and the resulting heat transfer. Thus, a finite volume method with immersed structure interfaces was implemented to study heat transfer through these media, with the aim of obtaining their ratio equivalent to fluid thermal conductivity (i.e. Nusselt number). Furthermore, our analysis shows that in such active wall constituted by two solid phases, under fixed weight constraints, the optimal value is difficult to predict a priori. Thus, different evolution laws of thermal gradient were studied, resulting in the change of the pore size distribution along some new anisotropic structures, in order to achieve the most one predictive.
\end{abstract}

\section{Nomenclature}

\section{Latin letters}

$\begin{array}{ll}\mathrm{N} & \text { buoyancy ratio, dimensionless } \\ \bar{m} & \text { arithmetic average, dimensionless } \\ f & \text { degree of freedom }\end{array}$

\section{Greek symbols}

$\epsilon \quad$ relative error, dimensionless

$\lambda$ thermal conductivity,

$\phi \quad$ heat flux, $W$

$\sigma \quad$ standard deviation, $\%$

$\theta \quad$ temperature, dimensionless

$\varepsilon \quad$ volume fraction, $\%$

Subscripts/Superscripts

eq equivalent value

$f \quad$ fluid phase

s solid phase

* $\quad$ solid to fluid ratio

\section{Introduction}

The study of transport phenomena in porous media of foam type is an increasingly active research field thanks to their large field of applications, the good control of their thermal and transfer characteristics, as well as their versatile compositions and architectures.

Metal foam structures were introduced by De Meller [1] and since the re-conception of their production methods made by Baumeister [2], the use of rigid foamtype porous media has been progressively increased thanks to their wide range of raw ingredients, including polymers, metals and ceramics (see more in [3]). Furthermore, much research has been performed in order to study these structures from different physical perspectives such as mechanical [4] and thermal [5].

These materials therefore often find applications as sinks or heat exchangers, because of their big surface area area and permeability and especially because these benefits can be coupled to the control over their morphology and composition, which allows a high degree of freedom in the design of models, improving properties like mechanical resistance and durability [6].

Previous works have presented the design of na active and reactive wall with change of interface through a porous structure of quasi-perfect insulation [7]. Besides, previous researches have demonstrated distinct responses for structures considered as homogeneous and inhomogeneous at the pore scale [8].

Focusing on the heat exchange advantages, and in order to achieve a more realistic model of termal transfers, several researches have shown that extended surfaces act as ideal effective sinks or heat exchangers thanks to their large surface areas to volume ratios which improve the heat transfer. Furthermore, numerous studies about heat exchangers with more regular geometries such as fins $[9,10]$ showed that the temperature gradient does not increase linearly along the longitudinal axis solicitation and it could present different optimal shapes following exponential or power functions that can maximize its heat transfer potential (see [11, Chap. 14-15] for more informations).

\footnotetext{
*Corresponding author: gisele.vieira@cefet-ri.br
} 
Thus, aiming to enrich the previously studied model of an active and reactive wall, this research will try to include this non-linear transfer behavior into a porous structure and to reach a quasi-perfect insulator through an inhomogeneous foam solid matrix composed by a solid phase and a void air-filled phase.

\section{Analysis and Modelling}

This section presents the generation process of one homogeneous and four inhomogeneous porous medium foam-type samples. Additionally, a numerical method used to characterize thermal properties will be presented.

Numerical foam-type structures generation: Firstly, one generates a homogeneous porous medium in two steps (Figure 1): generating a random porous structure based on correlated Gaussian random fields with a correlation length of $10 \%$, a standard deviation of $1 \%$, an expectation of 0 and 250 eigenmodes retained (a) and controlling its morphology by a threshold function (b).
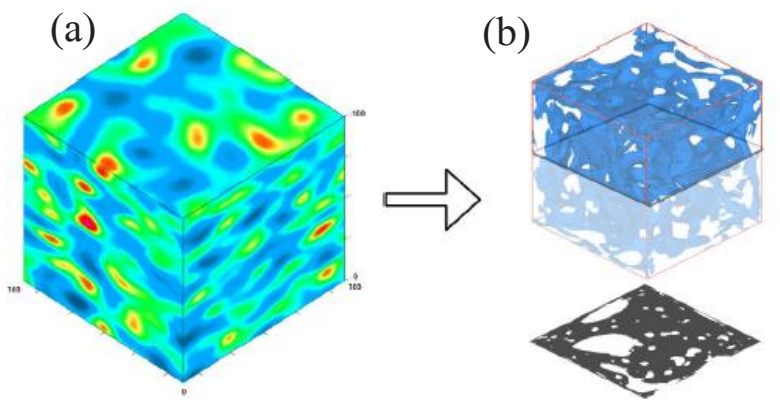

Fig. 1. (a) Correlated Gaussian random field; (b) phase partitioning threshold.

The homogeneity of this structure is verified in Table 1 which shows some properties and attest the small variability of its morphological componentes such as porosity, tortuosity and contact resistance.

Table 1. Morphology properties of homogeneous volume.

\begin{tabular}{|l|c|c|c|c|c|c|}
\cline { 2 - 7 } \multicolumn{1}{c|}{} & \multicolumn{2}{c|}{ Axis x } & \multicolumn{2}{c|}{ Axis y } & \multicolumn{2}{c|}{ Axis z } \\
\cline { 2 - 7 } \multicolumn{1}{c|}{} & $\bar{m}$ & $\sigma$ & $\bar{m}$ & $\sigma$ & $\bar{m}$ & $\sigma$ \\
\hline$\varepsilon_{\text {air }}$ & 87.17 & 2.5 & 87.17 & 3.14 & 87.17 & 4.05 \\
\hline Tort. & 1.50 & 0.15 & 1.25 & 0.11 & 1.47 & 0.18 \\
\hline Res. & 13 & - & 12 & - & 9 & - \\
\hline
\end{tabular}

Additionally, we generate the new inhomogeneous volumes based on the first homogeneous structure by applying a volume fraction gradient on the solid phase in the direction of two opposite sides, from the geometrical volume center. The Figure 2 present 4 steps of those transformations from $0 \%$ up to $\pm 20 \%$ following a linear function $f(x)=x$ with their respective thermal gradients. It should be noted that the transformation threshold applied in this work will be $20 \%(\mathrm{~d})$.
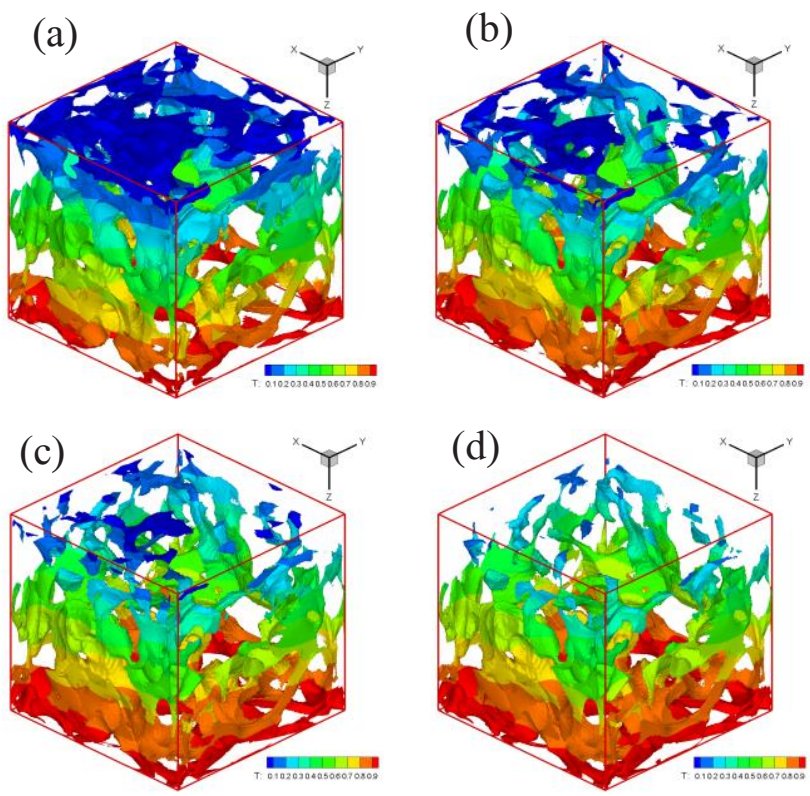

Fig. 2. (a) Homogeneous sample; inhomogeneous samples: (b) $10 \%$, (c) $15 \%$, (d) $20 \%$.

Then, four different gradient functions are applied as showed in Figure 3 in order to reach an optimal shape to the effective heat transfer properties.

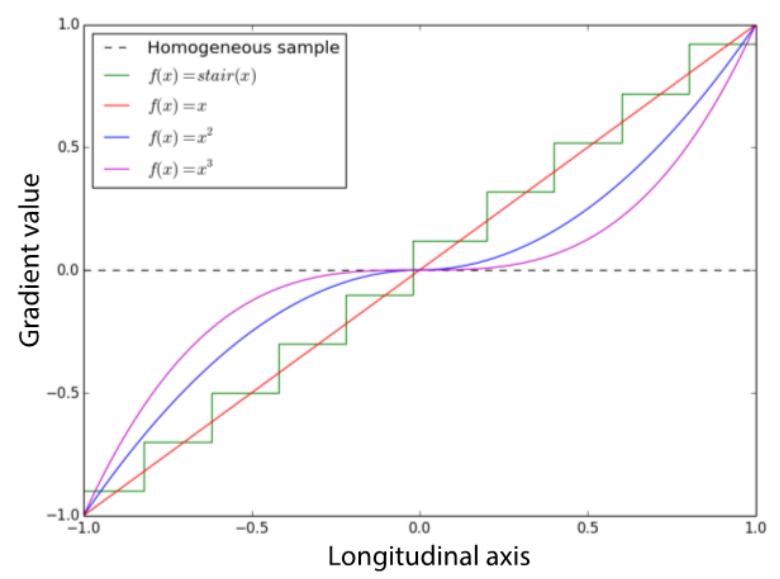

Fig. 3. Various implemented gradient functions.

Numerical method: Based on the finite volume method, a specially made device developed to study flows in porous media [12] through energy and momentum equations has been adopted in our study. This formulation takes into account, in the field of heat transfer, the conductive and convective transfers. Hence, the dimensionless equations are applied to the scheme configuration presented in Figure 4. 


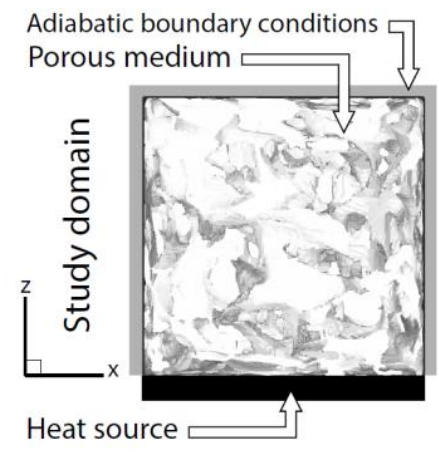

Fig. 4. Porous medium heat transfer configuration.

Thus, the complete formulation for a laminar mixed convection flow is written as follow.

Continuity equation :

$$
\nabla \overrightarrow{\mathrm{V}}=0
$$

Momentum equation:

$$
\begin{aligned}
\frac{\partial \overrightarrow{\mathrm{V}}}{\partial \mathrm{t}}+(\overrightarrow{\mathrm{V}} \cdot \vec{\nabla}) \overrightarrow{\mathrm{V}}=- & \vec{\nabla} \mathrm{P}+\frac{1}{\operatorname{Re}} \nabla\left(\mu^{*} \nabla \overrightarrow{\mathrm{V}}\right)+ \\
& \frac{1}{\mathrm{Da}^{*}} \overrightarrow{\mathrm{V}}+\frac{\mathrm{Ra}}{\operatorname{PrRe}^{2}}(\theta+\mathrm{NS}) \overrightarrow{\mathrm{k}}
\end{aligned}
$$

Energy equation:

$$
\frac{\partial \theta}{\partial \mathrm{t}}+\overrightarrow{\mathrm{V}} \cdot \nabla \theta=\frac{1}{\operatorname{Pr}} \nabla\left(\lambda^{*} \nabla \theta\right)
$$

The superscripted properties $\mu^{*}, D a^{*}$ and $\lambda^{*}$ are related to the heterogeneous material and will get the corresponding values as a function of the position within the heterogeneous material. Furthermore, for the interface boundary conditions, the continuity of the temperatures and of the normal heat flux vector is applied.

$$
\theta_{\mathrm{s}}=\theta_{\mathrm{f}}=\frac{\partial \theta_{\mathrm{f}}}{\partial \mathrm{n}}=\lambda^{*} \frac{\partial \theta_{\mathrm{s}}}{\partial \mathrm{n}}
$$

Hence, to reach a stable state solution, a convergence condition is implemented based on the temperature field.

$$
\frac{\sum_{\mathrm{i}, \mathrm{j}}\left|f_{\mathrm{i}, \mathrm{j}}^{\mathrm{m}+1}-f_{\mathrm{i}, \mathrm{j}}^{\mathrm{m}}\right|}{\sum_{\mathrm{i}, \mathrm{j}}\left|f_{\mathrm{i}, \mathrm{j}}^{\mathrm{m}+1}\right|} \leq \varepsilon=10^{-5}
$$

Where $\epsilon$ is the relative error, $m$ is an integer iteration counter, $\mathrm{f}$ is the degree of freedom of $\theta, P$ and $\mathrm{V}$. This method has presented good accuracy conforming to reference results published in preceding works [13]. Moreover, the validity criterion concerning this method is considered by $0.1 \leq \lambda^{*} \leq 200, R a=0$ and $P r=0.75$ with adiabatic domain boundary conditions for $\operatorname{Re}<<1$.
Furthermore, a validation test has been performed in order to compare our numerical model with analytical approach of homogenization [14] and another numerical approach method applied in [15]. This ratification is presented in Figure 5. The result confirms the consistency of the numerical solution as well as an accurate prediction of the equivalente diffusion characteristic.

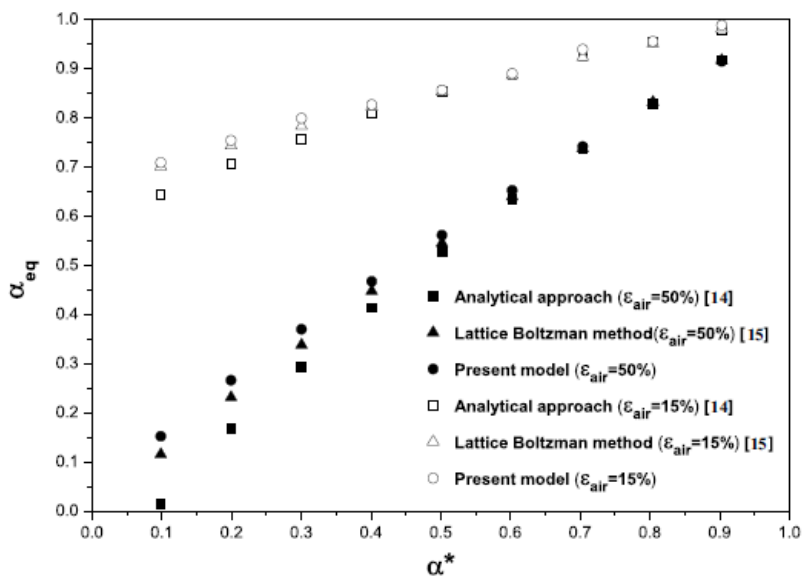

Fig.5. Numerical method validation.

\section{Results and Discussion}

Given the different threshold functions previously presented, this section will present their resulting samples with a morphological characterization as well as their porosimetry curves. To finish, a study of the equivalent thermal properties of each sample is set up in order to find the optimal shape configuration by means of thermal transfers.

Morphological characterization: In this part, one considers the functions presented in Figure 3 to demonstrate the generated inhomogeneous volumes after applying each different function. Figure 6 presents the void (fluid) phase distribution along the volume length which is quite regular for the homogeneous volume and rather irregular for the inhomogeneous volumes; the respective generated volumes are presented in Figure 7.

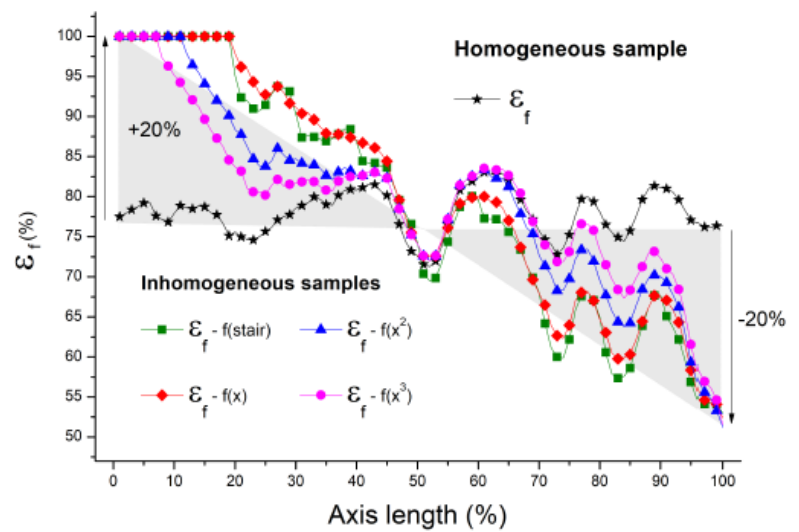

Fig.6. Volume fraction distribution along the solicitation axis. 


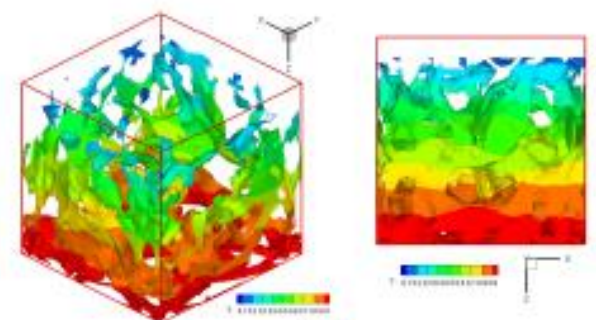

(a)
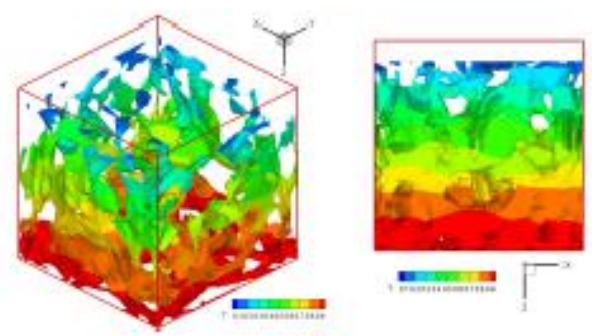

(b)
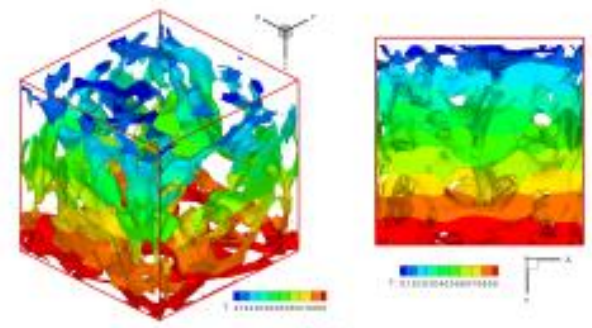

(c)

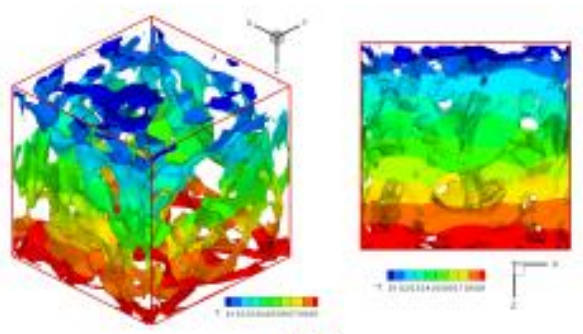

(d)

Fig.7. Inhomogeneous samples follow: (a) $f(x)=x$, (b) $f(x)=\operatorname{stair}(x),\left(\right.$ c) $f(x)=x^{2}$, (d) $f(x)=x^{3}$.

Table 2 gives some morphological properties of each sample. One observes that there is not a very important difference between the porosity values. However, the specific surface presents a notable variation with a most important rate for the function $f(x)=x^{3}$. These results are corroborated by Figures 6 and 7, where this model has the closest morphology properties to those of a homogeneous structure.

Table 2. Morphology properties of volumes.

\begin{tabular}{|c|c|c|}
\hline Sample & Porosity $\boldsymbol{\varepsilon}$ & Specific surface \\
\hline Homogeneous & $77.95 \%$ & $1.42 * 10^{5} \mathrm{~m}^{2} / \mathrm{m}^{3}$ \\
\hline$f(x)=x$ & $80.25 \%$ & $1.14 * 10^{5} \mathrm{~m}^{2} / \mathrm{m}^{3}$ \\
\hline$f(x)=$ stair $(x)$ & $79.31 \%$ & $1.17 * 10^{5} \mathrm{~m}^{2} / \mathrm{m}^{3}$ \\
\hline$f(x)=x^{2}$ & $79.68 \%$ & $1.24 * 10^{5} \mathrm{~m}^{2} / \mathrm{m}^{3}$ \\
\hline$f(x)=x^{3}$ & $79.57 \%$ & $1.29 * 10^{5} \mathrm{~m}^{2} / \mathrm{m}^{3}$ \\
\hline
\end{tabular}

Figure 8 describes, the pore size distribution (i.e. porosimetry) of the five generated volumes.

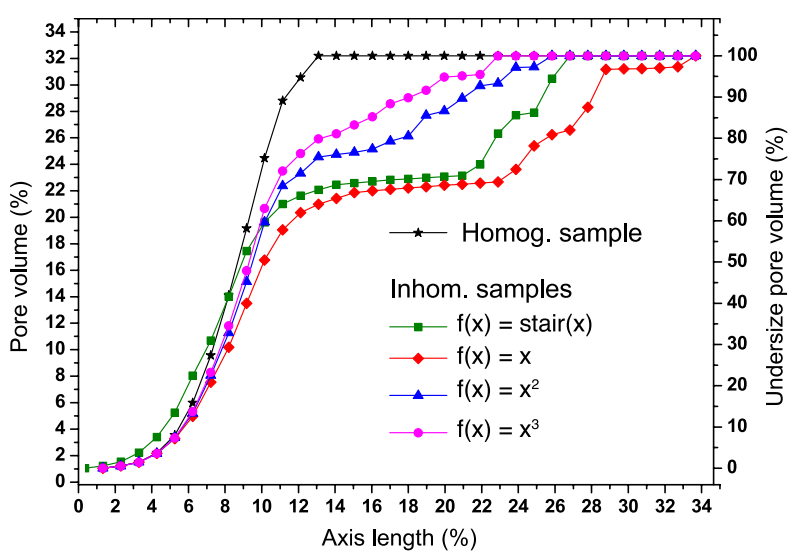

Fig.8. Void particle size distribution.

Indeed, it is quite clear that the objective of generating new structures of inhomogeneous morphology is achieved, especially observing the porosimetry distributions, where one can observe that the inhomogeneous structure shows a growth of the pore size range. However, besides the morphological properties of volumetric distribution, these structures will be further the source of different analyses and subsequent discussions with the purpose to verify the optimal shape function between the tested models.

Thermal characterization: Next, the equivalente thermal conductivity for each sample is characterized after heating on the lower side and with adiabatic boundary conditions for the other sides - Figure 4 . The equivalent properties of our samples are compared with several well-known approaches collected by Kaviany [16], modified by Bennacer et al. [17] and adapted for this study, in Figure 9.

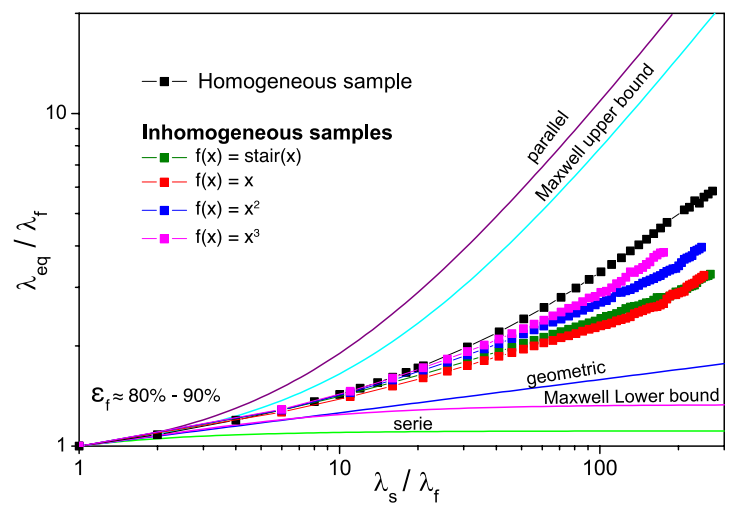

Fig.9. Ratios of thermal conductivity according to several approaches and the present model.

Considering that all approaches to compute equivalent properties will be placed between the parallel and series bounds, it is notable here that the calculated equivalent thermal conductivity of the homogeneous structure 
presents a result much closer to the upper bound than to the lower bound, which can be justified by its continuous solid phase and its consequences (e.g. percolation effect).

Otherwise, the inhomogeneous structures do not present the same behavior as the homogeneous. One observes that all configurations are less conductive than the first one. Nevertheless, the equivalent properties of each model follow the same proportionality to the specific surface, according to the information presented in Table 2. Moreover, the same as for the morphological analysis, the sample following the function $f(x)=x^{3}$ is also the nearest to the homogeneous sample, and it is finally the optimal shape in terms of thermal transfer properties.

\section{Conclusions}

In this study, we presented a morphological and thermal transfer characterization of some foam shaped porous media that could be employed, for example, as a superinsulator wall. The relevance of this description has been highlighted specially to understand the functioning of the non-linear thermal gradient present in these structures.

The generating procedure to create a homogeneous and various inhomogeneous volumes was proposed following different laws of thermal gradient, which results in the change of their pore size distributions. Additionally, the morphological characteristics accompanied by the porosimetry curve were presented and their thermal characterization showed that the optimum configuration among the inhomogeneous samples is the structure that follows the law $f(x)=x^{3}$, given its more important specific surface and equivalent thermal properties.

Finally, by understanding that the non-linear thermal problem is a major step towards the generation of an active and reactive structure in porous media foam-type, the presented results allow the opening up of new research opportunities and will be helpful to further investigate the abilities of multiphase porous media to manage their own equivalent permeability and conductivity. For numerical prediction purposes, this computational knowledge will be useful for more applications concerning the problems of heat transfer, air renewal, the coupling of those two and can even go beyond the domain of buildings.

Acknowledgments: We gratefully acknowledge the Laboratoire Mécanique et Technologie (LMTCachan) for the structure provided.

\section{References}

1. M.A. De Meller, Produit métallique pour l'obtention d'objets laminés, moulés ou autres, et procédés pour sa fabrication. (Patent FR 615147 A) (1926).

2. J. Baumeister, Method for producing porous metal bodies. (German Patent DE 4.018.360) (1990).
3. M. Ashby, T. Evans, N. Fleck, J. Hutchinson, H. Wadley, L. Gibson, Metal Foams: A Design Guide. Butterworth-Heinemann, Burlington (2000).

4. Q. Fang, J. Zhang, Y. Zhang, J. Liu, Z. Gong, Composite Structures 124, 409-420 (2015).

5. I. Ghosh, International Journal of Heat and Mass Transfer 52(5-6), 1488-1494 (2009).

6. K.S. Al-Athel, S.P. Aly, A.F.M. Arif, J. Mostaghimi, International Journal of Thermal Sciences 116(Supplement C), 199-213 (2017).

7. R.C. Deptulski, G.M.R. Vieira, R. Bennacer, International Conference on Materials \& Energy, Tiajin, China 1-6 (2017).

8. R.C. Deptulski, R. Bennacer, Journées Internationales de Thermique, Monastir, Tunisia 1-5 (2017).

9. R. Karvinen, T. Karvinen, International Journal of Heat and Mass Transfer 53(23), 5380 - 5385 (2010).

10. J.D. Osorio, A. Rivera-Alvarez, J.C. Ordonez, Applied Thermal Engineering 112, 572 - 584(2017).

11. A. Kraus, A. Aziz, J. Welty, 14 - Optimum design of radiating and convecting-radiating fins. In: Extended Surface Heat Transfer. Wiley (2001).

12. D. Mouhtadi, A. Amahmid, M. Hasnaoui, E.H. Kadri, R. Bennacer, Numerical Heat Transfer 65:1, 26-47 (2013).

13. A. AlAmiri, K. Khanafer, I. Pop, International Journal of Heat and Mass Transfer 52(15), 3818 3828 (2009).

14. T. Mori, K. Tanaka, Acta Metallurgica 21(5), 571574 (1973).

15. E. Walther, M. Bogdan, R. Bennacer, C.D. Sa, European Journal of Environmental and Civil Engineering 20(6), 667-679 (2016).

16. M. Kaviany, Principles of Heat Transfer in Porous Media. Mechanical engineering series. SpringerVerlag (1991).

17. R. Bennacer, K. Abahri, R. Belarbi, Woodhead Publishing Series in Civil and Structural Engineering. In: Intrinsic properties controlling the sustainability of construction. Second edition edn. Woodhead Publishing 33-53 (2016). 